\title{
Quantum Gravity and Phenomenological Philosophy ${ }^{1}$
}

\author{
Steven M. Rosen \\ College of Staten Island of the City University of New York
}

\begin{abstract}
The central thesis of this paper is that contemporary theoretical physics is grounded in philosophical presuppositions that make it difficult to effectively address the problems of subject-object interaction and discontinuity inherent to quantum gravity. The core objectivist assumption implicit in relativity theory and quantum mechanics is uncovered and we see that, in string theory, this assumption leads into contradiction. To address this challenge, a new philosophical foundation is proposed based on the phenomenology of Maurice Merleau-Ponty and Martin Heidegger. Then, through the application of qualitative topology and hypernumbers, phenomenological ideas about space, time, and dimension are brought into focus so as to provide specific solutions to the problems of force-field generation and unification. The phenomenological string theory that results speaks to the inconclusiveness of conventional string theory and resolves its core contradiction.
\end{abstract}

KEY WORDS: quantum gravity; phenomenology; philosophy; topology; string theory; dimension; subjectivity and objectivity

\section{INTRODUCTION}

Over two thousand years ago, Plato laid down a tripartite formula that would tacitly be incorporated into the foundations of modern science, and into Western culture at large. In a key passage of the Timaeus, he states: "we must make a threefold distinction and think of that which becomes, that in which it becomes, and the model which it resembles"(1) (p. 69). The first term refers to any particular object that is discernible through the senses. Plato speaks of the second term-"that in which [an object] becomes"- as the "receptacle," describing it as the vessel that contains the changing forms or objects without itself changing (p. 69). A little later, he characterizes this receptacle as space (pp. 71-72). Finally, the "model" for the transitory object is the "eternal object," the changeless form or archetype. This perfect form is eidos, a rational idea or ordering principle in the mind of the Demiurge. Using his archetypal thoughts as his blueprints,

\footnotetext{
${ }^{1}$ This article is published in Foundations of Physics, 2008, 38 (6), 556-82. The original publication is available at www.springerlink.com; information for citation by page number is also available from the author. The article is based on my 2008 book, The Self-Evolving Cosmos, appearing in the Series on Knots and Everything of World Scientific Publishing Company.
} 
the Divine Creator fashions an orderly world of particular objects and events. The third term brings in the element of subjectivity. Though the subject is divinely personified in the Timaeus, centuries later, with the Renaissance and the philosophy of René Descartes, subjectivity took on a more secular and general character. According to Descartes, whereas the object is res extensa, a thing extended in space, the subject is res cogitans, a "thinking thing" entirely without spatial extension. The threefold template thus adumbrated by Plato and carried forward in succeeding centuries is that of object-inspace-before-subject. $^{(2,3,4)}$ The object is what is experienced, the subject is the transcendent perspective from which the experience is had, and space is the continuous medium through which the experience occurs. The relationship among these three terms is that of categorical division.

By the time the Renaissance was over, the tripartite classical formula was firmly entrenched in human affairs and had assumed the status of a self-evident intuition. All human perception was now generally organized in terms objects appearing in space before the gaze of detached subjects. ${ }^{2}$ The formula is clearly in evidence in the domain of scientific analysis, where Descartes' philosophy is transposed into Cartesian mathematics via his system of coordinates. To see exactly how the formula applies, let us focus on a feature implicit in Plato's original account: while the object is transitory, the subject and the space in which it operates are not. This finds expression in all graphed equations. Consider the simple example of the equation for a parabola.

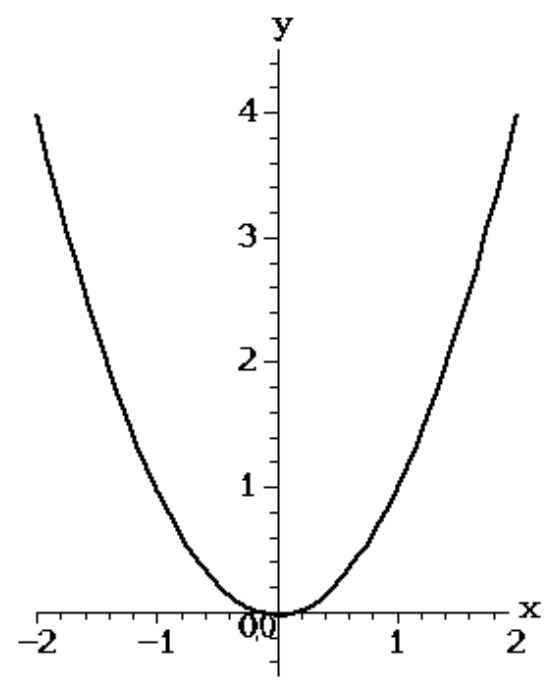

Figure 1. Graph of $y=x^{2}$, the equation for a parabola

In the graph of the equation (Fig. 1), $x$ and $y$ are variables, terms whose specific values change from point to point in the xy coordinate system. The variables correspond to the object term of the tripartite schema while the coordinate system itself constitutes the invariant spatial framework in which the objects are embedded. The third term of the

\footnotetext{
${ }^{2}$ Compare this with the mode of experience prevailing prior to the Renaissance, as intimated by philosopher Owen Barfield: "the world was more like a garment men [and women] wore about them than a stage on which they moved....Compared with us, they felt themselves and the objects around them and the words that expressed those objects, immersed together in something like a clear lake of... 'meaning"",(5) (p. 95).
} 
classical formula, viz. the subject, can also be located in the graph, at least indirectly. At the center of the xy coordinate system is the 0,0 origin. The origin of the function space plays a unique role. Representing the locus at which analysis or observation begins, 0,0 serves, in effect, as the surrogate for the subject's "eyes," for the fixed point of view from which empirical observations are made or analyses carried out (see Ref. 2, Chapter 1).

There is of course another key feature of the equation. By definition, the variables of the equation undergo change, but the relationship between these variables does not. The equation thus expresses what remains invariant when particular values in the coordinate system are transformed. The invariance of the equation mediates between the variability of the particular objects and the invariance of the subject and its space. We may say that the analytical subject brings order to the objects it encounters in space by imposing abstract invariance upon their concrete variability. Note that the equation's invariance clearly depends on the continuity of the functional space in which the relationship between variables is graphed. Were there a breach in the continuum, at this singular point the relationship would be abrogated and the equation would assume a nonfinite value. So whatever transformations of object-variables may occur within the spatial container, for the relationships between variables to be rendered invariant, the container itself must be invariant; it must stay intact, retain its continuity.

Though physics has been revolutionized since the days of Descartes, at bottom the old formula continues to operate. While the Michelson-Morley experiment indicated that Maxwell's equations for electromagnetism could not be shown to be invariant with respect to the classical dimensions of space and time, Einstein soon stepped in to demonstrate that the invariance of the equations could be established within a new and integrated framework of space-time. In special relativity, the equations for electromagnetic interaction remain invariant under global (Lorentzian) transformations of four-dimensional space-time coordinates; in general relativity, the equations for gravitational interaction are invariant under local (Riemannian) transformations of spacetime coordinates. Generally speaking then, relativity theory accounts for physical interactions by allowing the old space and time to vary within a new, more abstract context of changeless space-time.

As for the relationship between subject and object, it is instructive to compare pre-Einsteinian and Einsteinian cases. In the former, it was possible to assume a universal observer (a "Laplacean demon"). All objective events occurring in three-dimensional space were cast before the gaze of this idealized subject. What Einstein was able to demonstrate is that - when it comes to phenomena whose velocities approach the velocity of light - the local space and time of the concrete observer can no longer be discounted. Of course, Einstein did not simply accept the intrinsic variability of concrete observation. In his revision of classical physics, concrete observation itself is explicitly included in the account of nature by making this subjectivity into a new object, one whose transformations are invariant in a higher-dimensional context. So, with Einstein, the "objects" under scrutiny are now observational events transpiring in four-dimensional space-time. Whereas three-dimensional events are concretely observable, the fourth dimension of Einsteinian relativity is an abstraction. The higher-order Einsteinian observer of these four-dimensional acts of observation is therefore a further step removed from concrete reality than was his Cartesian predecessor. Nevertheless, in both cases, the traditional stance is strictly maintained. In both, we have object-in-space-before-subject. 
But doesn't the old formula break down in the other major revolution of modern physics? Does not the core discontinuity and accompanying subject-object interaction of quantum mechanics fly in the face of classical intuition? In the Copenhagen Interpretation of QM, a pragmatic approach is taken to this. No doubt the discontinuity lying at the heart of quantum theory entails a built-in limitation to the precision of objective measurement, yet uncertainty effects are readily manageable via probabilistic analysis as long as the phenomena under study are scaled well above the Planck length $\left(10^{-35}\right.$ meter). With this condition met, the classical formula can continue to operate as the default assumption. However, beginning in the last quarter of the twentieth century, the condition could no longer be met.

Before the 1970s, quantum physical research focused on the study of nongravitational forces (strong, weak, and electromagnetic) whose scales of operation exceeded the Planck length by a considerable margin. But as research progressed, physicists became more determined to arrive at a unified description of the seemingly diverse forces. Generally speaking, contemporary field theory associates the unification of nature's forces with a hotter, denser, microscopically scaled universe. The greater the number of forces to be unified, the higher the unification energy and the smaller the scale of magnitude at which the forces operate. In the 1970s, with electromagnetic and weak nuclear forces having been unified and advances made toward a grand unification of the three non-gravitational forces, physicists turned their attention to gravity. Now, in seeking to combine all four forces of nature in a single account, researchers could no longer avoid the ultra-microscopic Planck scale wherein uncertainty becomes uncontrollable and probabilistic analysis breaks down.

I venture to say that little significant progress has been made in the thirty years since the problem of quantum gravity first gained serious attention. To be sure, ambitious projects have been undertaken in this regard, most notably, string theory. This has been the dominant approach, generating prodigious amounts of mathematical research and much excitement in the theoretical physics community. I have offered a detailed critique of string theory elsewhere, ${ }^{(2,4)}$ and some of my own objections are reflected in two recent books on the subject. ${ }^{(6,7)}$ At present I will limit myself to addressing two major problems with the theory - one real and the other apparent.

According to string theory, the fundamental constituents of the physical universe are not zero-dimensional point particles as previously supposed (in the standard model of unification), but one-dimensional strings. The positing of fundamental vibrating strings is not motivated by empirical observation, nor is it based on positive theoretical insight as much as on a negative consideration. Whereas the supposition of vanishingly small point particles leads one to probe below the Planck length into the forbidden zone of unmanageable energies and nonsensical equations, the assumption of string-like elements whose finite extension is no smaller than the Planck length allows one to avoid such a pitfall. But while the general equations of string theory are expressly designed to eliminate the ambiguities of the trans-Planckian world, ambiguity is carried forward epistemically, making its presence felt in the vast multiplicity of possible solutions to those equations, with no provision of a guiding principle by means of which the field of possibilities can be reduced.

Beyond this problem, an apparent contradiction lies at the heart of string theory. We are going to see that, while this inconsistency at first may seem only to hammer 
another nail into string theory's coffin, in fact it may point toward the theory's redemption, albeit in a very different form.

\section{APPARENT SELF-CONTRADICTION OF STRING THEORY AND ITS PHILOSOPHICAL IMPLICATIONS}

Brian Greene emphasizes that "the spatially extended nature of a string is the crucial new element allowing for a single harmonious framework incorporating both [gravitational and quantum mechanical] theories" ${ }^{,(8)}$ (p. 136). But does the notion of a fundamental particle with finite extension make sense?

"Strings are truly fundamental" says Greene, "they are 'atoms,' uncuttable constituents" of nature. So, "even though strings have spatial extent, the question of their composition is without any content" ${ }^{(8)}$ (p. 141) Is there not a contradiction here? For-at least according to the classical concept of the continuum not explicitly challenged by string theory, to be an object extended in space is to be cuttable, in fact, infinitely divisible (see Refs. 2 and 4). How then could a string be a fundamental particle, an indivisible ingredient of nature, when it is spatially extended, thus divisible?! Let us look more closely at why string theory is led into such a contradiction for conventional thinking.

Within the classical framework of object-in-space-before-subject, the idea of an indivisible, point-like atom or particle is indispensable. In order for the subject to analyze effectively an object in space, s/he must be able to determine what the object is "made of," what its basic constituents are. There is a categorical difference between the atomic constituents of an object and the object itself. That is because, by definition, the "atoms" of which an object is composed, being indivisible, are not themselves open to analysis. The idea is not to say what they are made of; rather, they are the means by which the analysis of the object is performed. Therefore, the point-like elementary particles, instead of belonging with the object term of the classical formula, in fact belong with the subject term. The fundamental particle to which an object is reduced is the element upon which the subject's analysis of that object is built.

The standard model of unification maintains the classical assumption of point particles by adopting the attitude of positivistic pragmatism found in the Copenhagen Interpretation. Owing to the irreducible uncertainty inherent in Planck's constant, it is true that, the closer we come to the point-like atomic origin of observation and analysis, the fuzzier that origin becomes. Yet, in non-gravitational quantum mechanics, the fuzzing out of the origin is tolerable, since the uncertainty can be managed through probabilistic approximation. Because the calculations work quite well, says the Copenhagen Interpretation, there is no need to trouble ourselves with the meaning of the underlying uncertainty. What we don't know isn't hurting us in the practical activity of science that we engage in; therefore, we don't have to think about it.

For its part, string theory would also hope to effectively adopt a positivistic stance: we can't know what goes on below the fundamental Planck-scaled strings so we are entitled to ignore it. However, whereas the non-gravitational approach can afford its positivism, it seems that string theory cannot, since its positivism must be bought at the price of self-contradiction. 
Positivism becomes exorbitantly expensive when gravitation must be included in the unified account of nature. Given the colossal ultra-microscopic energies required for this when point particles are assumed, the Hilbert spaces used in probabilistic analysis collapse, with finite probability values no longer being assignable to terms that describe the physical states of particles. String theory's attempt to circumvent this problem and maintain positivism as a viable approach leads to the apparent contradiction we have discussed: an elementary particle with finite extension - that is, a point particle that is not a point particle. The particle cannot be a point if the ultra-microscopic infinities are to be avoided. And yet, the particle must be point-like, it must be indivisible, if an "objective" analysis is to be performed. For, if the particle is not elementary, if it does not have an unextended atomic character, analytical activity cannot be centered definitively in a point origin.

The apparent contradiction in string theory may be seen in terms of subject and object. Until the last quarter of the twentieth century, physics had done its utmost to suppress the fusion of subject and object intimated by QM's "problem of measurement." The standard model of unification had still been able to assume that the fundamental particle is simply unextended; therefore, the separation of subject and object could continue to be upheld, at least in probabilistic approximation: the point particle is the origin of the subject, and the extended entities that are observed and analyzed by the subject are its objects. Then, around 1975, when the need to deal with quantum gravity became a pressing issue, string theory entered the picture with its "extended atom." Unlike the point particle of standard unification theory, the string evidently must be both indivisible and spatially extended-in effect, both subject and object. It is this implication that subverts the posture of object-in-space-before-subject that physics has long sought to maintain in its idealized quest for unification.

Lee Smolin opens his recent book, The Trouble With Physics, by observing physics' current impasse: "For more than two centuries, until the present period [beginning roughly around 1975], our understanding of the laws of nature expanded rapidly. But today, despite our best efforts, what we know for certain about these laws is no more than what we knew back in the 1970s"(6) (p. viii). Smolin winds up calling for a different style of doing physics than what has been practiced since the advent of string theory. He advocates a "more reflective, risky, and philosophical style" (p. 294) that confronts "the deep philosophical and foundational issues in physics" (p. 290).

I applaud Smolin's call for a more philosophically-oriented physics. And I am proposing that the recent stalemate in physics suggests it will no longer be possible for us to rely on the old philosophical foundation. With the coming to prominence of the quantum gravity issue, theoretical physics evidently has reached an unprecedented watershed. The problems confronted by string theory, and by quantum gravity in general, are not merely theoretical ones that can be resolved within the extant philosophical framework of object-in-space-before-subject. Rather, the difficulty lies squarely with that framework itself. The trans-Planckian dissolution of spatial continuity and fusion of subject and object strike at the very heart of the ancient formula. I therefore venture to say that any new theory presupposing said formula will fail to bring the unification that is sought. But if the long-dominant tradition of philosophy is not equal to the task of 
effectively grounding an understanding of quantum gravity, is there any alternative philosophical foundation that can serve in this capacity? I believe the answer is yes.

\section{PHENOMENOLOGICAL PHILOSOPHY}

Science recognizes its pre-scientific roots. It rightly calls attention to the ignorance and confusion that prevailed before it came onto the scene; it correctly notes the benefits that were reaped with its emergence. But science also tends to believe that it has transcended history. While it readily acknowledges that its particular methods and findings are many and varied, that they are always subject to revision and refinement, science generally takes for granted that its posture of "objectivity" is beyond reproach. The assumption is that this detached stance gives us privileged access to the truth, and that it does so once and for all, exempting it from the possibility that there could be any constructive modification of it in the future. The uncritical acceptance of the "objective" posture (which is of course rooted in the ancient formula we have discussed) has made it difficult for science to address the problem of quantum gravity, since this problem - in intimating the trans-Planckian inseparability of subject and object-raises doubts about that posture. When confronted with the challenge of quantum gravity, most working scientists are not likely even to give serious attention to the question of science's basic epistemic position. And although a few courageous physicists like Lee Smolin have called for revolutionary new theories based on philosophical thinking, the default setting of philosophy remains operative, that of object-in-space-before-subject. What I am proposing is that meeting the challenge of quantum gravity requires that physics be regrounded in a new philosophy, one that can accommodate the intimate interplay of subject and object. I hasten to add that changing philosophy's "setting" in the way I am going to suggest will not mean losing contact with objective reality; on the contrary, it will mean that the analyst/observer, in relinquishing the stance of detached anonymity, will become more closely engaged with the down-to-earth facts of reality than ever before.

\subsection{Phenomenology}

Over the past century, the classical tradition has been questioned by the proponents of the philosophical initiative known as existential phenomenology. I will briefly describe the general features of this approach and its historical influence on physics, and will then focus on a specific concept in phenomenology that has an immediate bearing on the matter before us. We shall see that the idea of depth advanced by Maurice MerleauPonty ${ }^{(9)}$ responds to the challenge of quantum gravity by offering a new understanding of object, subject, and space.

The phenomenological movement is rooted in the nineteenth century existentialist writings of thinkers like Kierkegaard, Nietzsche, and Dostoevsky. It takes its contemporary form through the work of its principal figures: Edmund Husserl, Martin Heidegger, and Maurice Merleau-Ponty. In terms of the present article, phenomenology can be seen most essentially as a critique of the classical trichotomy of object-in-spacebefore-subject. To the phenomenologist, the activities of the detached Cartesian subject are idealizing objectifications of the world that conceal the concrete reality of the lifeworld. ${ }^{(10)}$ Obscured by the lofty abstractions of European science, this earthy realm of 
lived experience is inhabited by subjects that are not anonymous, that do not fly above the world, exerting their influence from afar. In the lifeworld, the subject is a fully situated, fully-fledged participant engaging in transactions so intimately entangling that it can no longer rightly be taken as separated either from its objects, or from the worldly context itself.

It is clear that all three terms of the classical formulation are affected by the phenomenological move. Again, on the classical account, the object is what is experienced, the subject is the transcendent perspective from which the experience is had, and space is the continuous medium through which the experience occurs. In this approach, objects are taken as simply external to each other and as appearing within a spatial continuum of sheer externality - space's infinite divisibility, or, in Heidegger's words, the "'outside-of-one-another" of the multiplicity of points"(11) (p. 481). The agents operating upon the objects entail a third mode of external relationship, acting as they do from a transcendent vantage point beyond the objects in space. It is this classical privileging of external relations that is counteracted in the phenomenological approach. Notwithstanding the Platonic/Cartesian idealization of the world, in the underlying lifeworld there is no object with boundaries so sharply defined that it is closed off completely from other objects. The lifeworld is characterized instead by the transpermeation of objects, by their mutual interpenetration, by the "reciprocal insertion and intertwining of one in the other," as Merleau-Ponty put it ${ }^{(12)}$ (p. 138). With objects thus related by way of mutual containment, no separate container is required to mediate their relations, as would have to be the case with externally related objects.

Phenomenological understanding supersedes the classical relationship of container and contained. Objects no longer are to be thought of as contained in space like things in a box, for, in containing each other, they contain themselves. At the same time, it must also be understood that, in the lifeworld, there can be no peremptory division of object and subject. The lifeworld subject, far from being the disengaged, high-flying deus ex machina of Descartes, finds itself down among the objects, is "one of the visibles"(12) ( $\mathrm{p}$. 135), is itself always an object to some other subject, so that the simple distinction between subject and object is confounded and "we no longer know which sees and which is seen" (p. 139). The phenomenological grounding of the subject is thus indicative of the close interplay of subject and object in the lifeworld. Generally speaking then, what the move from classical thinking to phenomenology essentially entails is an internalization of the relations among subject, object, and space.

Despite the continuing dominance of Cartesianism in contemporary physics, it is interesting to note that the phenomenology of Husserl and Heidegger did have an impact on some of the founders of quantum theory. Eugene Wigner, for example, had encountered Husserl at the University of Göttingen and knew Heidegger through Michael Polanyi, Wigner's colleague and mentor; these influences were reflected in Wigner's approach to physics (see Ref. 13). Another example is Werner Heisenberg's correspondence with Heidegger, which led Heisenberg to contribute an article on particle physics to a festschrift for Heidegger. ${ }^{(14)}$ The connection between phenomenology and physics is further evidenced in the work of scientists and philosophers such as Hermann Weyl, ${ }^{(15)}$ Joseph Kockelmans, ${ }^{(16)}$ and Pierre Kerszberg. ${ }^{(17)}$ A pivotal figure is Patrick Heelan, ${ }^{(18)}$ who studied with Wigner and Heisenberg, and has pioneered efforts to apply phenomenological investigation to the philosophy of space, and of quantum mechanics. 
Now, the beginnings of a specific phenomenological response to the problem of quantum gravity can be found in the work of Merleau-Ponty. ${ }^{(9)}$ In his concept of depth, he provides an account of dimensionality that permits us to understand the limitations of Cartesian space and to surpass them. I will describe Merleau-Ponty's approach in the following section, then attempt to clarify it in the section after that.

\subsection{Depth: The Primary Dimension}

By way of introducing Merleau-Ponty's depth dimension, let us consider once more the traditional dichotomy between objects contained in space and their spatial container, or, as Plato put it, between "that which becomes [and] that in which it becomes"(1) (p. 69). This approach to space ultimately devitalizes the world, renders it immobile, because, whatever changes may transpire in the objects that "become," however they may be transformed, the containing space, the Platonic receptacle, itself does not change (p. 69). Indeed, for there to be change, there must be difference, contrast, opposition of some kind. But the point-elements that make up the classical continuum, rather than entailing opposition, involve mere juxtaposition. Being unextended and thus devoid of inner structure, the elements of space possess no gradations of depth, no shading, texture, or nuance, no contrasts or distinctions of any sort. Instead of expressing the interplay of shadow and light, space itself is all light, as it were. A condition of "total exposure" prevails for the point-elements of the continuum, since these elements, having no interior recesses, must be said to exist solely "on the outside." All that could be said of the relations among such eviscerated beings is what Heidegger said: the points of classical space are "“outside-of-one-another'." So, rather than actively engaging each other as do the beings that are contained in space, the densely packed elements of the classical container sit inertly side by side, like identical beads on a string.

As a matter of fact, even though the beings that dwell in such a space can be said to be "actively engaged," the quality of their interaction is affected by the context in which they are embedded: since the continuum is constituted by sheer externality, the relations among its inhabitants must also be external. Classical dynamics are essentially mechanistic; instead of involving a full-fledged dialectic of opposition and identity wherein beings influence each other from core to core, influence is exerted in a more superficial fashion. According to David Bohm, the mechanistic order of influence is one in which entities "interact through forces that do not bring about any changes in their essential natures ... [they interact] only through some kind of external contact"(19) (p. 173). We may say then that classical space contains dialectical process in such a way that it externalizes it, divesting it of its depth and vitality.

In Merleau-Ponty's phenomenological approach, we see that the classical space appearing to contain dialectical process actually originates from it. In his essay "Eye and Mind," Merleau-Ponty emphasizes the "absolute positivity" of traditional Cartesian space $^{(9)}$ (p. 173). For Descartes, space simply is there; possessing no folds or nuances, it is the utterly explicit openness, the sheer positive extension that constitutes the field of strictly external relations wherein unambiguous measurements can be made. Classical space, says Merleau-Ponty, "remains absolutely in itself, everywhere equal to itself, homogeneous; its dimensions, for example, are interchangeable (p. 173)." He concludes 
that, for Descartes, space is a purely "positive being, outside all points of view, beyond all latency and all depth, having no true thickness" (p. 174).

Challenging the Cartesian view, Merleau-Ponty insists that the dialectical features of perceptual experience (perspectival opposition, gradations of depth, etc.) are not merely secondary to a space that itself is devoid of such features. He begins his own account of spatiality by exploring the paradoxical interplay of the visible and invisible, of difference and identity, that is characteristic of true depth:

The enigma consists in the fact that I see things, each one in its place, precisely because they eclipse one another, and that they are rivals before my sight precisely because each one is in its own place. Their exteriority is known in their envelopment and their mutual dependence in their autonomy. Once depth is understood in this way, we can no longer call it a third dimension. In the first place, if it were a dimension, it would be the first one; there are forms and definite planes only if it is stipulated how far from me their different parts are. But a first dimension that contains all the others is no longer a dimension, at least in the ordinary sense of a certain relationship according to which we make measurements. Depth thus understood is, rather, the experience of the reversibility of dimensions, of a global 'locality' - everything in the same place at the same time, a locality from which height, width, and depth [the classical dimensions] are abstracted. ${ }^{(9)}$ (p. 180)

Speaking in the same vein, Merleau-Ponty characterizes depth as "a single dimensionality, a polymorphous Being," from which the Cartesian dimensions of linear extension derive, and "which justifies all [Cartesian dimensions] without being fully expressed by any" (p. 174). The dimension of depth is "both natal space and matrix of every other existing space" (p. 176).

Merleau-Ponty goes on to observe that primal dimensionality must be understood as self-containing. This is illustrated through the visual space of Cézanne: "Cézanne knows already what cubism will repeat: that the external form, the envelope, is secondary and derived, that it is not that which causes a thing to take form, that this shell of space must be shattered" (p. 180). In breaking the "shell," one disrupts the classical representation of objects-in-space. Now space is no longer taken in abstraction from its content, but in recognition of the unbroken flow from container to content. "We must seek space and its content as together," says Merleau-Ponty (p. 180). It is in this sense of the internal mediation of container and content that Cézanne's depth dimension is selfcontaining.

It is also clear that this primal dimension engages embodied subjectivity: the dimension of depth "goes toward things from, as starting point, this body to which I myself am fastened" (p. 173). In his comment that "there are forms and definite planes only if it is stipulated how far from me their different parts are" (p. 180; italics mine), Merleau-Ponty is conveying the same idea. And a little later, Merleau-Ponty says:

The painter's vision is not a view upon the outside, a merely "physical-optical" relation with the world. The world no longer stands before him through representation; rather, it is the painter to whom the things of the world give birth 
by a sort of concentration or coming-to-itself of the visible. Ultimately the painting relates to nothing at all among experienced things unless it is first of all 'autofigurative.'...The spectacle is first of all a spectacle of itself before it is a spectacle of something outside of it. (p. 181)

In this passage, the painting of which Merleau-Ponty speaks, in drawing upon the originary dimension of depth, draws in upon itself. Painting of this kind is not merely a signification of objects but a self-signification that surpasses the division between object and subject.

In sum, the phenomenological dimension of depth as described by Merleau-Ponty is (1) the "first" dimension, inasmuch as it is the source of the Cartesian dimensions, which are idealizations of it; it is (2) a self-containing dimension, not merely a container for contents that are taken as separate from it; and it is (3) a dimension that leads back to and brings into play the actions of the lived subject, rather than serving as but a staging platform for the detached operations of an anonymous subject. In other words, depth constitutes the dimensional structure of the lifeworld, the primal world obscured by the abstractions of classical experience. In realizing depth, we go beyond the concept of space as but an inert container and come to understand it as an aspect of an indivisible cycle of action in which the "contained" and "uncontained" — object and subject — are integrally incorporated.

\section{TOPOLOGICAL PHENOMENOLOGY}

Merleau-Ponty's concept of depth surpasses the ancient formula by internalizing the relations among object, space, and subject. Whereas the Platonic-Cartesian intuition of these ontological categories makes it impossible to come to grips with the discontinuity and intimate subject-object interaction of the trans-Planckian domain, depth-dimensional intuition is better attuned to the quantum gravitational realm. However, it is obvious that a great gap exists between the softly-focused phenomenological notion of depth and the more sharply defined concepts and phenomena of theoretical physics. The remainder of this paper is dedicated to closing this gap.

The bridge I propose for joining seemingly distant shores is topological phenomenology (see Refs. 2, 3, and 4). To conventional thinking, topology is generally defined as the branch of mathematics that concerns itself with the properties of geometric figures that stay the same when the figures are stretched or deformed. In algebraic topology, structures from abstract algebra are employed to study topological spaces. A more concrete approach to topology is exemplified by the practical experiments of mathematician Stephen Barr. ${ }^{(20)}$ In either case, however, the underlying philosophical default setting tacitly operates, with topological structures regarded strictly as objects under the scrutiny of a detached analyst. Yet, in Heidegger's enigmatic invocation of a "topology of Being"(21) (p. 12), and in Merleau-Ponty's reference to "topological space as...constitutive of life" ${ }^{(12)}$ (p. 211), there is a first intimation of a phenomenologicallybased, non-objectifying topology. As a matter of fact, when Merleau-Ponty metaphorically describes this topological space as "the image of a being that...is older than everything and 'of the first day"' (p. 210), we are reminded of the concept of 
dimension he had outlined in his earlier work: the concept of depth. ${ }^{(9)}$ Can we sharpen our focus on the depth dimension by going further with topology? A well-known topological curiosity appears especially promising in this regard: the Klein bottle.

Elsewhere, I have used the Klein bottle to address a variety of philosophical issues ${ }^{(22,23,24)}$ (see also Refs. 2 and 3). For our present purpose, we begin with a simple illustration.

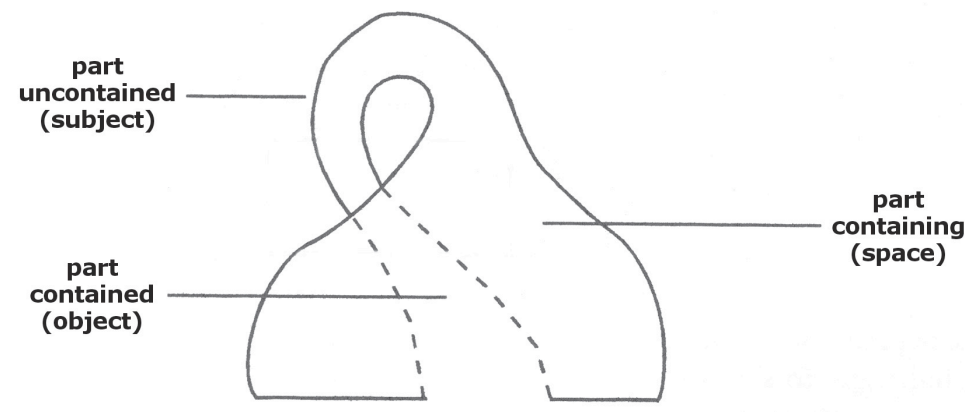

Figure 2. Parts of the Klein bottle (after Ryan, Ref. 25, p. 98)

Figure 2 is my adaptation of communication theorist Paul Ryan's linear schemata for the Klein bottle ${ }^{(25)}$ (p. 98). According to Ryan, the three basic features of the Klein bottle are "part contained," "part uncontained," and "part containing." Here we see how the part contained opens out (at the bottom of the figure) to form the perimeter of the container, and how this, in turn, passes over into the uncontained aspect (in the upper portion of Fig. 2). The three parts of this structure thus flow into one another in a continuous, self-containing movement that flies in the face of the classical trichotomy of contained, containing, and uncontained - symbolically, of object, space, and subject. But we can also see an aspect of discontinuity in the diagram. At the juncture where the part uncontained passes into the part contained, the structure must intersect itself. Would this not break the figure open, rendering it simply discontinuous? While this is indeed the case for a Klein bottle conceived as an object in ordinary space, the true Klein bottle actually enacts a dialectic of continuity and discontinuity, as will become clearer in further exploring this peculiar structure. We can say then that, in its highly schematic way, the one-dimensional diagram lays out symbolically the basic terms involved in the "continuously discontinuous" dialectic of depth. Depicted here is the process by which the three-dimensional object of the lifeworld, in the act of containing itself, is transformed into the subject. This blueprint for phenomenological interrelatedness gives us a graphic indication of how the mutually exclusive categories of classical thought are surpassed by a threefold relation of mutual inclusion. It is this relation that is expressed in the primal dimension of depth.

When Merleau-Ponty says that the "enigma [of depth] consists in the fact that I see things...precisely because they eclipse one another," that "their exteriority is known in their envelopment," he is saying, in effect, that the peremptory division between the inside and outside of things is superseded in the depth dimension. Just this supersession is embodied by the Klein bottle. What makes this topological surface so surprising from the classical standpoint is its property of one-sidedness. More commonplace topological figures such as the sphere and the torus are two-sided; their opposing sides can be identified in a straightforward, unambiguous fashion. Therefore, they meet the classical 
expectation of being closed structures, structures whose interior regions ("parts contained") remain interior. In the contrasting case of the Klein bottle, inside and outside are freely reversible. Thus, while the Klein bottle is not simply an open structure, neither is it simply closed, as are the sphere and the torus. In studying the properties of the Klein bottle, we are led to a conclusion that is paradoxical from the classical viewpoint: this structure is both open and closed. The Klein bottle therefore helps to convey something of the sense of dimensional depth that is lost to us when the fluid lifeworld relationships between inside and outside, closure and openness, continuity and discontinuity, are overshadowed in the Cartesian experience of their categorical separation.

However, must the self-containing one-sidedness of the Klein bottle be seen as involving the spatial container? Granting the Klein bottle's symbolic value, could we not view its inside-out flow from "part contained" to "part containing" merely as a characteristic of an object that itself is simply "inside" of space, with space continuing to play the classical role of that which contains without being contained? In other words, despite its suggestive quality, does the Klein bottle not lend itself to classical idealization as a mere object-in-space just as much as any other structure?

A well-known example of a one-sided topological structure that indeed can be treated as simply contained in three-dimensional space is the Moebius strip. Although its opposing sides do flow into each other, this is classically interpretable as but a global property of the surface, a feature that depends on the way in which the surface is enclosed in space but one that has no bearing on the closure of space as such; that is, the topological structure of the Moebius, the particular way its boundaries are formed (one end of the strip must be twisted before joining it to the other), can be seen as unrelated to the sheer boundedness constituted by the structureless point elements of space itself. So, despite the one-sidedness of the Moebius strip, the three-dimensional space in which it is embedded can be taken as retaining its simple closure. The maintenance of a strict distinction between the global properties of a topological structure and the local structurelessness of its spatial context is mathematics' way of upholding the underlying classical relation of object-in-space. Given that the Moebius strip does lend itself to drawing said categorical distinction, can we say the same of the Klein bottle? Although conventional mathematics answers this question in the affirmative, I will suggest the contrary.

The schematic representation of the Klein bottle provided by Figure 2 shows that it possesses the odd property of passing through itself. When we consider the actual construction of a Klein bottle in three-dimensional space (by joining one boundary circle of a cylinder to the other from the inside), we are confronted with the fact that no structure can penetrate itself without cutting a hole in its surface, an act that would render the model topologically imperfect (simply discontinuous). So the Klein bottle cannot be effectively assembled when one is limited to three dimensions.

Mathematicians observe that a form that penetrates itself in a given number of dimensions can be produced without cutting a hole if an added dimension is available. The point is imaginatively illustrated by Rudolf Rucker. ${ }^{(26)}$ He asks us to picture a species of "Flatlanders" attempting to assemble a Moebius strip, which is a lower-dimensional analogue of the Klein bottle. Rucker shows that, since the reality of these creatures would be limited to two dimensions, when they would try to make an actual model of the Moebius, they would be forced to cut a hole in it. Of course, no such problem with 
Moebius construction arises for us human beings, who have full access to three external dimensions. It is the making of the Klein bottle that is problematic for us, requiring as it would a fourth dimension. Try as we might we find no fourth dimension in which to execute this operation.

However, in contemporary mathematics, the fact that we cannot create a proper model of the Klein bottle in three-dimensional space is not seen as an obstacle. The modern mathematician does not limit him- or herself to the concrete reality of space but feels free to invoke any number of higher dimensions. Notice though, that in summoning into being these extra dimensions, the mathematician is extrapolating from the known three-dimensionality of the concrete world. This procedure of dimensional proliferation is an act of abstraction that presupposes that the nature of dimensionality itself is left unchanged. In the case of the Klein bottle, the "fourth dimension" required to complete its formation remains an extensive continuum, though this "higher space" is acknowledged as but a formal construct; the Klein bottle per se is regarded as an abstract mathematical object simply contained in this hyperspace (whereas the sphere, torus, and Moebius strip are relatively concrete mathematical objects, since tangibly perceptible models of them may be successfully fashioned in three dimensions). We see here how the conventional analysis of the Klein bottle unswervingly adheres to the classical formulation of object-in-space. Moreover, whether a mathematical object must be approached through hyperdimensional abstraction or it is concretizable, the mathematician's attention is always directed outward toward an object, toward that which is cast before his or her subjectivity. This is the aspect of the classical stance that takes subjectivity as the detached position from which all objects are viewed (or, better perhaps, from which all is viewed as object); here, never is subjectivity as such opened to view. Thus the posture of contemporary mathematics is faithfully aligned with that of Plato and Descartes in whatever topic it may be addressing. Always, there is the mathematical object (a geometric form or algebraic function), the space in which the object is contained, and the seldom-acknowledged uncontained subjectivity of the mathematician who is carrying out the analysis.

Now, in his study of topology, Barr advised that we should not be intimidated by the "higher mathematician.... We must not be put off because he is interested only in the higher abstractions: we have an equal right to be interested in the tangible"(20) (p. 20). The tangible fact about the Klein bottle that is glossed over in the higher abstractions of modern mathematics is its hole. Because the standard approach has always presupposed extensive continuity, it cannot come to terms with the inherent discontinuity of the Klein bottle created by its self-intersection. Therefore, all too quickly, "higher" mathematics circumvents this concrete hole by an act of abstraction in which the Klein bottle is treated as a properly closed object embedded in a hyper-dimensional continuum. Also implicit in the mainstream approach is the detached subjectivity of the mathematician before whom the object is cast. I suggest that, by staying with the hole, we may bring into question the classical intuition of object-in-space-before-subject.

Let us look more closely at the hole in the Klein bottle. This loss in continuity is necessary. One certainly could make a hole in the Moebius strip, torus, or any other object in three-dimensional space, but such discontinuities would not be necessary inasmuch as these objects could be properly assembled in space without rupturing them. It is clear that whether such objects are cut open or left intact, the closure of the space 
containing them will not be brought into question; in rendering these objects discontinuous, we do not affect the assumption that the space in which they are embedded is simply continuous. With the Klein bottle it is different. Its discontinuity does speak to the supposed continuity of three-dimensional space itself, for the necessity of the hole in the bottle indicates that space is unable to contain the bottle the way ordinary objects appear containable. We know that if the Kleinian "object" is properly to be closed, assembled without merely tearing a hole in it, an "added dimension" is required. Thus, for the Klein bottle to be accommodated, it seems the three-dimensional continuum itself must in some way be opened up, its continuity opened to challenge. Of course, we could attempt to sidestep the challenge by a continuity-maintaining act of abstraction, as in the standard mathematical analysis of the Klein bottle. Assuming we do not employ this stratagem, what conclusion are we led to regarding the "higher" dimension that is required for the completion of the Klein bottle? If it is not an extensive continuum, what sort of dimension is it? I suggest that it is none other than the dimension of depth adumbrated by Merleau-Ponty.

Depth is not a "higher" dimension or an "extra" dimension; it is not a fourth dimension that transcends classical three-dimensionality. Rather-as the "first dimension"(9) (p. 180), depth constitutes the dynamic source of the Cartesian dimensions, their "natal space and matrix" (p. 176). Therefore, in realizing depth, we do not move away from classical experience but move back into its ground where we can gain a sense of the primordial process that first gives rise to it. The depth dimension does not complete the Klein bottle by adding anything to it. Rather, the Klein bottle reaches completion when we cease viewing it as an object-in-space and recognize it as the embodiment of depth. It is the Kleinian pattern of action (as schematically laid out in Fig. 2) that expresses the in-depth relations among object, space, and subject from which the old trichotomy is abstracted as an idealization. So it turns out that, far from the Klein bottle requiring a classical dimension for its completion, it is classical dimensionality that is completed by the Klein bottle, since - in its capacity as the embodiment of depth-the Klein bottle exposes the hitherto concealed ground of classical dimensionality. Here is the key to transforming our understanding of the Klein bottle so that we no longer view it as an imperfectly formed object in classical space but as the dynamic ground of that space: we must recognize that the hole in the bottle is a hole in classical space itself, a discontinuity that, when accepted dialectically instead of evaded, leads us beyond the concept of dimension as continuum to the idea of dimension as depth.

By way of summarizing the paradoxical features of the Klein bottle thus far considered, I refocus on the threefold disjunction implicit in the standard treatment of the Klein bottle: contained object, containing space, uncontained subject. (1) The contained constitutes the category of the bounded or finite, that of the immanent contents we reflect upon, whatever they may be. These include empirical facts and their generalizations, which may be given in the form of equations, invariances, or symmetries. (2) The containing space is the contextual boundedness serving as the means by which reflection occurs. (3) The uncontained or unbounded is the transcendent agent of reflection, namely, the subject. It is in adhering to this classical trichotomy that the Klein bottle is conventionally deemed a topological object embedded in "four-dimensional space." But the actual nature of the Klein bottle suggests otherwise. The concrete necessity of its hole indicates that, in reality, this bottle is not a mere object, not simply enclosed in a 
continuum as can be assumed of ordinary objects, and not opened to the view of a subject that itself is detached, unviewed (uncontained). Instead of being contained in space, the Klein bottle may be described as containing itself, thereby superseding the dichotomy of container and contained. Instead of being reflected upon by a subject that itself remains out of reach, we may say that the self-containing Kleinian object is self-reflexive: it flows back into the subject thereby disclosing - not a detached cogito, but the dimension of depth that constitutes the dialectical lifeworld.

\section{THE PHYSICAL SIGNIFICANCE OF THE KLEIN BOTTLE}

Having fleshed out Merleau-Ponty's depth dimension via Kleinian topology, we are now prepared to consider the physical meaning of the phenomenologically constituted Klein bottle. The characteristics of Kleinian depth we have examined bring to mind the intimate subject-object interaction and discontinuity found at the core of quantum physics. I believe this makes it reasonable to entertain the idea that the Kleinian action structure embodies $\hbar$, the quantized action associated with the emission of radiant energy in the microworld. In point of fact, this connection is already implicit in the standard formulation of subatomic spin, though the relationship is well disguised.

Subatomic particles are endowed with internal angular momentum or spin. According to Roger Penrose, ${ }^{(27)}$ spin is the "most obvious physical concept that one has to start with, where quantum mechanics says something is discrete" (p. 151). The fundamental quantized action of spin, indexed by $\hbar / 2$, hardly takes the form of a simply continuous spinning in three-dimensional space. When Wolfgang Pauli sought to model quantum mechanical spin, he employed the mathematics of complex numbers, and, in particular, the "hypernumbers" developed by William Clifford in the nineteenth century (see Ref. 28). Mathematician David Applebaum observes that Clifford's abstract algebraic research had been "motivated by geometry...particularly the problem of trying to generalize the properties of complex numbers so as to be able to describe rotations in three dimensions"(29) (p. 3). Pauli used the "simplest non-trivial example of a Clifford algebra" (p. 4) to derive three matrices, which yield the three components of electron spin:

$$
\begin{aligned}
& S_{x}=\hbar / 2\left(\begin{array}{llll}
0 & 1 & 1 & 0
\end{array}\right) \\
& S_{y}=\hbar / 2\left(\begin{array}{llll}
0 & -i & i & 0
\end{array}\right) \\
& S_{z}=\hbar / 2\left(\begin{array}{llll}
1 & 0 & 0 & -1
\end{array}\right),
\end{aligned}
$$

where $\hbar / 2$ is the basic unit of electron spin. In fact, $\hbar / 2$ is taken as the basis for determining the spin of all subatomic particles. Fermions, which are typically matter particles like the electron and quark, have spin values that are odd-number multiples of $\hbar / 2$ (such as $1 \hbar / 2,3 \hbar / 2,5 \hbar / 2$, etc.). Bosons, which are typically force particles like the photon or gluon, entail spin values that are even-number multiples of $\hbar / 2$ (such as $2 \hbar / 2$, $4 \hbar / 2$, etc.).

Note that, while the Pauli spin matrices employ the hypernumber $i$, they are based on a form of hypernumber that actually goes beyond $i$. The mathematician Charles Musès called this number $\varepsilon$, defined as $\varepsilon^{2}=+1$, but $\varepsilon \neq \pm 1$. Musès associated each of Pauli's 
three matrices with a different variety of $\varepsilon$ (Ref. 30, p. 213). But Musès was not satisfied with a merely algebraic expression of $\varepsilon$. Emphasizing the intimate relationship between algebra and geometry, he asserted that "geometry...can lead us to a deeper understanding" than the mere "brute facts" of algebra:

The hypernumber $i$ needs only a plane for visualization because, as it is multiplied by itself, it rotates in a plane as does a unit radius when a circle is drawn. But the hypernumber $\varepsilon$ involves reflection....It is clear that in order to turn a right hand into its reflected version (a left hand), more is required than sliding or rotation in tri-dimensional space. It can be shown that a right hand would be changed into a left hand if it were rotated $180^{\circ}$ out of our triple dimensional space into fourdimensional space, and then back into our space again....Therefore, because $\varepsilon$ deals with reflections and not only with simple rotations as does $i$, its operations demand geometrically a four-dimensional space, whereas the simple, rotational character of $i$ 's operations demands only two-dimensional space. ${ }^{(28)}$ (p. 77)

Musès further observes that when the "kinematic geometry of epsilon" is applied to physics, it becomes "clear that [electron] spin is more like successive rotation through a four-dimensional space and then back into ours, rather than an ordinary, threedimensional spinning. As of this writing, it is realized among physicists that such 'spin' is not ordinary; but $\varepsilon$ clarifies what it is" (p. 77).

Elsewhere $^{(22)}$ (p. 104), I related the kinematic geometry of $\varepsilon$ to the topology of the Klein bottle. Like $\varepsilon$ rotation, Kleinian action can also be seen as involving a "successive rotation through a four-dimensional space and then back into ours"; moreover, the Klein bottle possesses the property of reflection that transforms left into right and vice versa. I would maintain, of course, that the "extra dimension" into which Kleinian action flows is in fact no objectified fourth dimension but the sub-objective dimension of depth. This is the crucial distinction between the proposed phenomenological approach to the foundations of physics and the still-prevalent Cartesian one.

It might not be too much to say that all microworld dynamics arise from spin of the Kleinian kind $(\varepsilon \hbar / 2)$. The fundamental role of subatomic spin is brought out by $\mathrm{F}$. A. M. Frescura and Basil Hiley. ${ }^{(31)}$ These theorists associate spin with "the pre-geometric structure of the holomovement" (p. 27) - the dynamic substrate that Bohm portrayed as underlying space, time, and quantum mechanics. ${ }^{(19) 3}$ The significance of spin is also highlighted in an article by David Hestenes entitled, "Quantum Mechanics from SelfInteraction." "(32) According to Hestenes, electromagnetic self-interaction is the core element of quantum mechanics, and spin is its "most characteristic feature" (p. 68). In emphasizing the central importance of spin, Hestenes indicates that the uncertainty relations $\left(\Delta x \Delta p_{x}=\hbar / 2\right)$ found in every aspect of quantum theory derive from spin: "We now see the uncertainty relations as consequences of a zero-point motion with a fixed zero-point angular momentum, the spin of the electron. This explains why the limiting constant $\hbar / 2$ in the uncertainty relations is exactly equal to the magnitude of the electron spin" (p. 73).

\footnotetext{
${ }^{3}$ The notion of an underlying pattern of movement more primordial than space and time and giving rise to them is reminiscent of Leibniz' idea that the space-time world is built up from spaceless and timeless action centers called "monads."
} 
Citing Hestenes and others, Huping $\mathrm{Hu}$ and Maoxin $\mathrm{Wu}^{(33)}$ venture considerably further in arguing for the fundamental role played by spin in physics and beyond. Their basic proposition is well summarized in the title of their article: "Spin as Primordial SelfReferential Process Driving Quantum Mechanics, Spacetime Dynamics and Consciousness." Hu and Wu thus do not limit themselves to the claim that spin is the source of physics. Primordial spin is "protopsychic" (p. 45) as well as protophysical; it is "the seat of consciousness and the linchpin between mind and brain" (p. 43). Or we may say that spin is psychophysical or sub-objective, not just an objective event taking place in physical space or a subjective happening enclosed within the psyche. Moreover, $\mathrm{Hu}$ and $\mathrm{Wu}$, in portraying the self-referential nature of spin, come close to recognizing its Kleinian character. Associating spin with the particle self-interaction of which Hestenes speaks, they liken it to the recursive "strange loops" described by Douglas Hofstadter. Here there is "an interaction between levels in which the top level reaches back down towards the bottom level influencing it, while at the same time being itself determined by the bottom level" (p. 45). What we find when we turn to Hofstadter himself is that the "strange loop" may be related to the Klein bottle ${ }^{(34)}$ (p. 691). Evidently then, quantized microphysical process is rooted in Kleinian spatio-sub-objectivity.

\section{PHENOMENOLOGICAL STRING THEORY AND QUANTUM GRAVITY}

\subsection{The Depth-Dimensional "String Quartet"}

Recall the apparent self-contradiction underlying string theory discussed in Section 2: Planck-scaled strings are implicitly both indivisible and spatially extended, thus divisible; in effect, they are both subject and object. Yet an unacceptable contradiction when considered from the trichotomizing perspective of classical thinking can become a fact of dialectical process when grasped phenomenologically. If we take the vibratory pattern of the fundamental strings as essentially Kleinian in nature-with Kleinian spin not objectified but understood in its phenomenological depth — string theory can gain greater coherence. In fact, by reformulating the theory in the context of topological phenomenology, not only can the theory's central contradiction be resolved, but, also, its current inconclusiveness can be addressed. As noted in Section 1, the quantum gravitational equations of string theory lead to a vast multiplicity of possible solutions with no guiding principle by means of which the field can be narrowed. What I demonstrate elsewhere ${ }^{(4)}$ is that string theory can be cast in a phenomenological form that provides a detailed and definitive (albeit qualitative) account of quantum gravity, one that unambiguously yields the fundamental particles of the standard model. In this introductory paper, I will take the liberty of limiting myself to a summary of these findings.

In his further exploration of the hypernumber $\varepsilon$, Musès indicated a "higher epsilonalgebra" wherein " $\sqrt{ } \varepsilon_{\mathrm{n}}$ involves $i_{\mathrm{n}}$, the subscripts of course referring to the $(n+1)$ th dimension since $i \equiv i_{1}$ already refers to $\mathrm{D}_{2}{ }^{\prime(35)}$ (p. 42). Bearing in mind the intimate relationship between $\varepsilon$ and the Klein bottle, can Musès' implication of a dimensional 
hierarchy of hypernumber values be given topo-phenomenological expression? The Klein bottle does lend itself to such a generalization.

Mathematicians have investigated the transformations that result from bisecting topological surfaces. If the Klein bottle is bisected, cut down the middle, it will fall into a pair of oppositely-oriented Moebius strips. Next, bisecting the one-sided Moebius strip, a two-sided lemniscatory surface will be produced, its sides being related enantiomorphically (i.e., as mirror opposites). Finally, cutting the lemniscate down the middle yields interlocking lemniscates. The transformation brought about by this bisection is clearly the last one of any significance, since additional bisections - being bisections of lemniscates, can only produce the same results: interlocking lemniscates. The bisection series is completed then when we obtain interlocking lemniscates, a structure we shall designate the sub-lemniscate. By thus experimenting with the bisection of the Klein bottle, we discover a nested family of four topological structures.

Of course, in such a bisection experiment, we work with a tangible model of the Klein bottle, implicitly taking it as but an object in space. Operating in this fashion, the topological structures unfolded via bisection all manifest as two-dimensional surfaces. The bisection series thus gives no direct evidence of the hierarchy of differently dimensioned $\varepsilon$-like spin structures intimated by Musès. Yet we do know that there is a dimensional difference between Kleinian and Moebial structures. We are aware that, whereas an imperfect model of the Klein bottle can be constructed in three-dimensional space, a properly formed bottle would require an "added" dimension to fill its hole. The dimension in question is no transcendent "fourth" dimension but the dimension of depth that completes the Klein bottle as a spatio-sub-objective lifeworld. Unlike the Klein bottle, the Moebius surface can successfully be assembled as an object in threedimensional space. Rucker's thought experiment ${ }^{(26)}$ has told us that it would be in twodimensional space that the Moebius could not be properly put together without producing a hole. Evidently then, in the two-dimensional milieu the Moebius would play the same role as played by the Klein bottle in three-dimensional space. What this suggests is thateven though the Moebius appears as but an object in space to the three-dimensional observer, in its own two-dimensional sphere it would function as a depth dimension, as a spatio-sub-objective lifeworld unto itself. Similar conclusions can be reached about the other two members of the bisection series: the lemniscate would constitute the onedimensional lifeworld and the sub-lemniscate the zero-dimensional lifeworld. It is this account of several different topodimensional lifeworlds nested within each other that is consistent with the hierarchy of $\varepsilon$-like spin structures adumbrated by Musès.

\begin{tabular}{|c|c|c|c|}
\hline$\varepsilon_{\mathrm{D} 0}$ & $\varepsilon_{\mathrm{D} 0} / \varepsilon_{\mathrm{D} 1}$ & $\varepsilon_{\mathrm{D} 0} / \varepsilon_{\mathrm{D} 2}$ & $\varepsilon_{\mathrm{D} 0} / \varepsilon_{\mathrm{D} 3}$ \\
\hline$\varepsilon_{\mathrm{D} 1} / \varepsilon_{\mathrm{D} 0}$ & $\varepsilon_{\mathrm{D} 1}$ & $\varepsilon_{\mathrm{D} 1} / \varepsilon_{\mathrm{D} 2}$ & $\varepsilon_{\mathrm{D} 1} / \varepsilon_{\mathrm{D} 3}$ \\
\hline$\varepsilon_{\mathrm{D} 2} / \varepsilon_{\mathrm{D} 0}$ & $\varepsilon_{\mathrm{D} 2} / \varepsilon_{\mathrm{D} 1}$ & $\varepsilon_{\mathrm{D} 2}$ & $\varepsilon_{\mathrm{D} 2} / \varepsilon_{\mathrm{D} 3}$ \\
\hline$\varepsilon_{\mathrm{D} 3} / \varepsilon_{\mathrm{D} 0}$ & $\varepsilon_{\mathrm{D} 3} / \varepsilon_{\mathrm{D} 1}$ & $\varepsilon_{\mathrm{D} 3} / \varepsilon_{\mathrm{D} 2}$ & $\varepsilon_{\mathrm{D} 3}$ \\
\hline
\end{tabular}

Table 1. Interrelational matrix of topodimensional spin structures

Table 1, the topodimensional spin matrix, gives the $\varepsilon$-based counterpart of our topological bisection series. The three-dimensional Kleinian spinor is written $\varepsilon_{\mathrm{D} 3}$, with lower-dimensional members of the tightly knit spin family designated $\varepsilon_{\mathrm{D} 2}, \varepsilon_{\mathrm{D} 1}$, and $\varepsilon_{\mathrm{D} 0}$ (corresponding to the Moebial, lemniscatory, and sub-lemniscatory circulations, 
respectively). These terms are arrayed on the principal diagonal of the matrix (the one extending from the upper left-hand corner to the lower right). The interrelationships among the four principal matrix elements, taken two at a time, are reflected in the elements appearing off the main diagonal. We may better understand the role of the offdiagonal terms by employing a musical analogy.

Generally speaking, Table 1 unpacks the dialectical structure of topodimensional interrelations. Regarding topodimensional action as inherently vibratory in nature, we note the similarity of this table to the old Pythagorean table (Table 2).

\begin{tabular}{|l|l|l|l|}
\hline $1 / 1$ & $1 / 2$ & $1 / 3$ & $1 / 4$ \\
\hline $2 / 1$ & $2 / 2$ & $2 / 3$ & $2 / 4$ \\
\hline $3 / 1$ & $3 / 2$ & $3 / 3$ & $3 / 4$ \\
\hline $4 / 1$ & $4 / 2$ & $4 / 3$ & $4 / 4$ \\
\hline
\end{tabular}

Table 2. Section of the Pythagorean table

The Pythagorean table is usually portrayed as an indefinitely expanding series of musical intervals. What is shown in the limited section of the table that I have selected for illustrative purposes is a set of relationships that essentially corresponds to our topodimensional action matrix: there is a principal diagonal that contains a series of fundamental vibrations or tones, and these four principal intervals are coupled to each other two at a time by six pairs of overtone-undertone intervals related to each other in the mirror-opposed fashion of enantiomorphs. (The overtone ratios are the $>1$ values extending below the fundamental tones, whereas the undertone ratios are the $<1$ values appearing to the right of the fundamentals.)

Consider in Table 1 the two principal "tones" of highest dimensionality: $\varepsilon_{\mathrm{D} 2}$ and $\varepsilon_{\mathrm{D} 3}$. These matrix elements are linked by the "overtone" and "undertone" given in the two corresponding non-principal cells, $\varepsilon_{\mathrm{D} 3} / \varepsilon_{\mathrm{D} 2}$ and $\varepsilon_{\mathrm{D} 2} / \varepsilon_{\mathrm{D} 3}$ (respectively). The enantiomorphically-related coupling cells in question are the depth-dimensional counterparts of the concretely observable, oppositely oriented Moebius strips which, when glued together, form the Klein bottle. Taken strictly as a principal matrix element, the depth-dimensional Moebius vibration is the spin structure that constitutes the twodimensional lifeworld $\left(\varepsilon_{\mathrm{D} 2}\right)$. But when we shift our view of the Moebius, consider it in relation to higher, Kleinian dimensionality, a kind of "doubling" takes place in which the $\varepsilon_{\mathrm{D} 2}$ singular Moebius spin structure becomes a pair of asymmetric, mirrored opposed twins, $\varepsilon_{\mathrm{D} 3} / \varepsilon_{\mathrm{D} 2}$ and $\varepsilon_{\mathrm{D} 2} / \varepsilon_{\mathrm{D} 3}$. It is through the fusion of these dimensional enantiomorphs that Kleinian dimensionality is crystallized. Since the Table-1 matrix indicates that all four principal dimensionalities or fundamental tones are interrelated by accompanying off-diagonal overtone-undertone pairs, we can draw the general conclusion that higher dimensions emerge through processes of enantiomorphic fusion (this is fully detailed in Ref. 4). 
The process of dimensional generation can be clarified in broad terms by relating it to a reverse movement through the bisection series wherein topological structures are not divided but glued together. To begin, we imagine the fusion of interlocking lemniscates that yields the single lemniscate. This corresponds to the generation of the one-dimensional lifeworld $\left(\varepsilon_{\mathrm{D} 1}\right)$. Next, we picture the enantiomorphically-related sides of the two-sided lemniscate merging to form the one-sided Moebius structure, this being associated with the genesis of the two-dimensional lifeworld $\left(\varepsilon_{\mathrm{D} 2}\right)$. Finally, we imagine Moebius enantiomorphs fusing to produce the Klein bottle, which corresponds to the evolution of our three-dimensional lifeworld $\left(\varepsilon_{\mathrm{D} 3}\right)$. With each fusion, a lowerdimensional lifeworld is introjected by a higher-dimensional one, incorporated in such a way that the lower dimension is concealed. In the end, we have three lower-dimensional vibratory structures concealed within the three-dimensional Kleinian vibration, much as lower dimensions are hidden by becoming "curled up" within visible $3+1$-dimensional space-time in the conventional string theoretic account of dimensional cosmogony. It turns out, in fact, that the phenomenological approach arrives at the same total number of dimensions as does the conventional theory. To see how this is so, let us consider more closely the dimension of time.

In the course of questioning contemporary efforts to arrive at a workable theory of quantum gravity, Lee Smolin made this confession: "there is something basic we [physicists] are all missing, some wrong assumption we are all making"(6) (p. 256). Smolin goes on to say:

I strongly suspect that the key is time....Time is represented as if it were another dimension of space. Motion is frozen, and a whole history of constant motion and change is presented to us as something static and unchanging.... We have to find a way to unfreeze time - to represent time without turning it into space. (pp. 25657)

I venture to say that regrounding theoretical physics in phenomenological philosophy has the effect of "unfreezing time." Merleau-Ponty's depth dimension is not simply a dimension of space, nor is it even a domain of space-time, in the Einsteinian sense in which time is essentially spatialized. A better term for the lifeworld dimension is "timespace." This was Heidegger's name for the dynamic order of being integrating time and space in such a way that the rule of fixed spatiality is surpassed in favor of a more authentic, "unfrozen" temporality (what Heidegger also called "true time"). ${ }^{(36)}$ The close kinship of time-space with depth is elaborated elsewhere (Ref. 4, pp. 52-53). Here I only want to emphasize that, once the world is brought to life by recognizing the dialectical process that underlies it, this lifeworld is no longer mistaken as constituted by static spatiality but is indeed understood as a time-space. Perhaps we might say then the Kleinian lifeworld is not a three- or four-dimensional space nor a $3+1$-dimensional space-time, but a 1+3-dimensional time-space. And nested within this lifeworld are three others: the $1+2$-dimensional Moebial time-space, the 1+1-dimensional lemniscatory time-space, and the 1+0-dimensional sub-lemniscatory time-space. Such an account, however, would not be entirely accurate. 
The time and space of the lifeworld are blended more intimately than in the classical continuum, making it problematic to view their relationship as simply additive $(1+0,1+1$, etc.). Yet, in Section 3.2, we did find that classical space-time originates from the more primordial spatiotemporality of the depth dimension (what Merleau-Ponty regarded as "natal space and matrix of every other existing space"). So while it may not be much more accurate to describe the Kleinian lifeworld as "1 + 3-dimensional" than as "3 + 1-dimensional," we can and should say that 3 + 1-dimensional space-time is projected from primordial Kleinian time-space $\left(\varepsilon_{\mathrm{D} 3}\right)$. The other three space-time regimes would similarly be projected from their three time-space counterparts (Moebial, lemniscatory, and sub-lemniscatory). A simple summation of projected space-time dimensions gives us a total of ten, with the six lower dimensions- $-(2+1)+(1+1)+(0$ $+1)$-being hidden like Russian dolls within the larger $3+1$-dimensional space-time. This picture of overall ten-dimensionality, with six dimensions concealed, accords with the basic account provided by string theory. Thus we may say that our four temporospatial spinors spin out the ten ${ }^{4}$ space-time dimensions of string theory.

By way of underscoring the inherent musicality of this depth-dimensional "string quartet," I end this section with a quote. According to Brian Greene:

Music has long...provided the metaphors of choice for those puzzling over questions of cosmic concern. From the ancient Pythagorean "music of the spheres" to the "harmonies of nature" that have guided inquiry through the ages, we have collectively sought the song of nature in the gentle wanderings of celestial bodies and the riotous fulminations of subatomic particles. With the discovery of superstring theory, musical metaphors take on a startling reality, for the theory suggests that the microscopic landscape is suffused with tiny strings whose vibrational patterns orchestrate the evolution of the cosmos. ${ }^{(8)}$ (p. 135)

\subsection{The Music of Evolving Nature}

String theorists have approached cosmogony by adopting the concept of symmetry breaking. According to the prevailing view, the four forces of nature are conceived as vibrating strings that initially existed in a purely symmetric ten-dimensional space scaled around the Planck length. Subsequently, the perfect primordial symmetry was spontaneously broken by a dimensional bifurcation in which four of the original dimensions expanded to produce the visible universe we know today, with the other dimensions remaining hidden. Coupled with this was the breaking of force-field symmetry to create the appearance of irreconcilable differences among the forces.

However, while the foregoing account of cosmogony incorporates both dimensional and force-field symmetry breaking, the two are not precisely aligned with each other in the theoretical reckoning. This reflects the fact that contemporary theorists have been unable to articulate a detailed geometric rendering of cosmic evolution. Heinz Pagels begins his discussion of the extra-dimensional (Kaluza-Klein) interpretation of cosmogony on an optimistic note: "Remarkably, the local gauge symmetries are precisely

\footnotetext{
${ }^{4}$ With the extension of string theory known as $M$-theory, eleven dimensions are actually entailed, though the eleventh dimension is not like the other ten. This "extra" dimension in fact may be interpreted as intimating the depth dimension. See Ref. 4, Chapter 8.
} 
the symmetries of the compact higher-dimensional space. Because of this mathematical fact, all the gauge theories of Yang-Mills fields can be interpreted purely geometrically in terms of such compact higher-dimensional spaces"(37) (pp. 327-28). And yet, for the geometric program fully to be realized, the physical events described in the standard and inflationary models of cosmic development would need to be specifically expressible as dimensional events. "Unfortunately," admits Pagels, "no one has yet been able to find a realistic Kaluza-Klein theory which yields the standard model" (p. 328). In the stringtheoretic application of Kaluza-Klein theory, one obvious reason for this limitation is the absence of a conceptual principle that could guide the analyst to unambiguous solutions of the ten-dimensional general equations (see Section 1), solutions specifying the exact shapes of the hidden dimensions that would correspond to the physical facts of the standard model. Of course, if the prevailing theory cannot tell us what the dimensional structures are that correspond to physical reality, it can hardly inform us on how these dimensions develop. In point of fact, there is really no positive feature intrinsic to the theory that provides for the evolution of dimensions. As far as I can tell, the only reason dimensional bifurcation is assumed to have taken place at all is that theorists must somehow account for the present inability to observe six of the ten dimensions needed for a consistent rendering of quantum gravity (one that avoids untenable probability values).

Smolin seems to put his finger on the underlying problem in calling attention to the "wrong assumption" physicists "are all making" when they present the "whole history of constant motion and change... as something static and unchanging"(6) (pp. 256-57). When authentic change is thus denied, it is not surprising that no natural, parsimonious way of accounting for cosmogony is forthcoming. Conventional string theory well exemplifies this adherence to the classical intuition of changelessness in the primacy it gives to the notion of symmetry. It is in assuming an initial state of "perfect symmetry" that theorists must resort to the artifice of "spontaneous symmetry breaking," an alleged event that — far from being a natural consequence of the purely symmetric theory - is gratuitously invoked without a compelling explanation of its basis.

Phenomenological string theory affords a way out of the impasse. Here time is "unfrozen." Instead of artificially appending asymmetry to a primordially perfect symmetry, a dialectic of symmetry and asymmetry is offered that permits an unequivocal, intrinsically meaningful account of the evolving forces of nature. This principle of "synsymmetry" $(3,4,22)$ is implicit in the topological bisection series and its associated topodimensional spin matrix (Table 1).

For a simple illustration, consider the Moebius strip. It arises from the fusion of mirror-opposed, asymmetrically-related sides of the lemniscate. We can say that, through this union of opposites, the asymmetry of lemniscatory sides is rendered symmetric. However, while the Moebius can be deemed symmetric vis-à-vis the fused lemniscatory sides that constitute it, at the same time it is itself a member of an enantiomorphic pair whose own fusion produces the Klein bottle. Generally speaking, we may conclude that the members of our topodimensional family are neither simply asymmetric nor simply symmetric, but synsymmetric: a given member combines symmetry and asymmetry in such a way that it is symmetric in relation to its lower-dimensional counterpart and asymmetric in relation to its higher one (the sub-lemniscate is an exception to this, since it has no lower-dimensional counterpart). I propose that the synsymmetry concept, viewed dynamically in terms of enantiomorphic fusion events, constitutes a guiding 
principle for cosmogony. The forces and particles of nature evolve by a general process wherein asymmetric dimensional enantiomorphs fuse to create a dimensional symmetry that at once inherently gives way to new asymmetry. To keep this article at a manageable length, I will restrict myself to a synoptic sketch of topo-phenomenological cosmogony (see Ref. 4 for more detail).

What I am suggesting is that a full account of the elementary forces of string theory may be afforded by embedding the theory in the matrix of primordial spin structures given in Table 1. The matrix in question constitutes a special application of the Clifford-based hypernumber idea that provides a highly specific rendition of primordial spin action, a topodimensional array of four fundamental spinors (displayed on the principal diagonal of the matrix) that can be directly associated with the four types of gauge bosons found in nature. The gauge-boson correlates of Table 1 are given in Table 3 . What is the basis of these correlations?

\begin{tabular}{|c|c|c|c|}
\hline $\mathrm{G}$ & $\mathrm{G} / \mathrm{g}$ & $\mathrm{G} /(\mathrm{W}, \mathrm{Z})$ & $\mathrm{G} / \gamma$ \\
\hline $\mathrm{g} / \mathrm{G}$ & $\mathrm{g}$ & $\mathrm{g} /(\mathrm{W}, \mathrm{Z})$ & $\mathrm{g} / \gamma$ \\
\hline$(\mathrm{W}, \mathrm{Z}) / \mathrm{G}$ & $(\mathrm{W}, \mathrm{Z}) / \mathrm{g}$ & $\mathrm{W}, \mathrm{Z}$ & $(\mathrm{W}, \mathrm{Z}) / \gamma$ \\
\hline$\gamma / \mathrm{G}$ & $\gamma / \mathrm{g}$ & $\gamma /(\mathrm{W}, \mathrm{Z})$ & $\gamma$ \\
\hline
\end{tabular}

Table 3. Spin matrix of gauge bosons. $\mathrm{G}$ is the graviton; $\mathrm{g}$ is the strong gauge boson; $\mathrm{W}, \mathrm{Z}$ is the weak gauge boson particle pair; and $\gamma$ is the photon

We know that Table 1 signifies a process of generation in which higher topological dimensions evolve from lower ones. The facts of physical evolution lend themselves to straightforward, one-to-one correlation with topogenetic process. The first force particle to "freeze out" of the Big Bang's hot primordial soup is the hypothesized graviton, $\mathrm{G}$. The graviton of Table 3 is associated with $\varepsilon_{\mathrm{D} 0}$, the zero-dimensional sublemniscatory action of Table 1 , which can be written $\varepsilon_{\mathrm{D} 0}(\hbar / 2)$ to give expression to subatomic particle spin; thus, $\mathrm{G} \equiv \varepsilon_{\mathrm{D} 0}(\hbar / 2)$. Next to separate itself from the primordial chaos is the strong gauge boson, $\mathrm{g}$, and we relate it to $\varepsilon_{\mathrm{D} 1}$ lemniscatory action, writing $\mathrm{g} \equiv$ $\varepsilon_{\mathrm{D} 1}(\hbar / 2)$. Then the weak force emerges, given by the boson pair $\mathrm{W}$ and $\mathrm{Z}$, which we identify with $\varepsilon_{\mathrm{D} 2}(\hbar / 2)$. When the three orders of lower-dimensional gauge boson have "frozen out," what remains is $\gamma$, the photon, topodimensionally expressed as $\varepsilon_{\mathrm{D} 3}(\hbar / 2)$.

Having focused our attention on the principal terms or "fundamental tones" of our matrices, let us now inquire into the physical significance of the "overtone-undertone" couplings appearing off the principal diagonals. In Table 1, these are the topodimensional enantiomorphs whose synsymmetric fusions drive the process of dimensional generation. The overtone-undertone couplings appear in Table 3 as enantiomorphically-related boson ratios. It is from their interactions that the primary gauge bosons emerge. Since nature's force fields evolve by a process in which the universe expands, boson-ratio fusion may be regarded as impelling said expansion. I conjecture accordingly that these primordial boson ratio interactions, which are not themselves directly observable, comprise the mysterious "dark energy" said to fuel the accelerated expansion of the cosmos.

In phenomenological string theory, boson-ratio interaction not only accounts for the generation of the four kinds of gauge bosons, but for the production of the 12 
fermions of the standard model as well. The six pairs of ratios involved in distilling the bosons also interact to yield the six pairs of fermions (three lepton pairs and three quark pairs). Geometrically speaking, the fermions function as "dimensional bounding elements," local features of global bosonic dimensionality, with local and global aspects intimately interwoven (in keeping with Merleau-Ponty's notion of the depth dimension as a "global locality"; see Section 3). Needless to say, this requires clarification, but I do not have the space to elaborate further on it here (see Ref. 4). I will only suggest that the purely geometric account of boson-fermion interrelatedness I am proposing obviates the need for the unparsimonious and unsubstantiated postulation of particle "super-partners" given in the currently influential notion of "supersymmetry."

\section{EPISTEMOLOGICAL POSTSCRIPT: TOWARD A NEW KIND OF CLARITY}

In the preceding pages, I have attempted to demonstrate the advantages of grounding quantum gravity in phenomenological philosophy. Of significant benefit is the provision of a detailed and definitive account of dimensional generation, one that brings string theory down to earth by aligning it unambiguously with the qualitative facts of the standard model of particle physics. Another unique feature of the new approach is its clarification of dimensions with respect to whether they are space-like or time-like. Whereas conventional string/Kaluza-Klein theory is vague on this score, the phenomenological account identifies exactly four orders of spatiotemporality containing a total of six space-like dimensions and four time-like ones. Most importantly perhaps, phenomenological string theory meets Smolin's requirement of unfreezing time.

However, comparing conventional and phenomenological approaches to quantum gravity has its pitfalls. It is easy to underestimate the magnitude of the change that is entailed in making the transition from one to the other. Does the phenomenological version of string theory really clarify the conventional rendition by providing more detail on dimensional patterning, as I have claimed? In general, I believe the answer is yes, but it might actually be more accurate to say that phenomenology offers a new kind of clarity, one in which the idea of "detail" has a rather different meaning.

Assuming the conventional posture of mathematical physics, we begin from an intuition of abstract universality, viz. the symmetric forms that constitute the general equations. ("Physicists...believe [their] theories are on the right track because, in some hard-to-describe way, they feel right, and ideas of symmetry are essential to this feeling"; Ref. 8, p. 225). We then look to solve those equations so as to determine the particular structures that can be put into correspondence with physical reality. However, the general and particular levels of the theory are separated from each other in such a way that the sense of intuitive confidence felt about the abstract symmetries does not carry over into the detailed solutions of the equations. That is why conventional string theory has had such difficulty determining which of its many possible topological solutions is the right solution. Physicists have had to play the elaborate guessing games we have discussed because their mathematical formulations afford no intuitive guidance as to the right shape of the hidden dimensions. The "devil" surely has been in the details.

In the contrasting phenomenological approach, the generalities intuited are inseparable from the details, since these general structures are not abstractions that must 
be concretized in a subsequent step but are "general things" (12) (p. 139), universals that themselves are concrete. So, when I say that the phenomenological version of KaluzaKlein theory gives more detail on dimensional patterning than the conventional version, I am not speaking of "details" in the usual sense of particular features or instances of a pattern that itself is more general, the division of the particular and general being tacitly assumed. Rather, I am referring to a pattern whose details are an integral part of its very generality. This surely makes for a different kind of clarity than is customarily sought. I submit, however, that, in confronting the profound challenge of quantum gravity, the customary manner of clarification is simply not equal to the task. It is here that phenomenology can play its crucial role.

\section{REFERENCES}

1. Plato, Timaeus and Critias, D. Lee, trans. (Penguin, New York, 1965).

2. S. M. Rosen, Dimensions of Apeiron (Editions Rodopi, New York and Amsterdam, 2004).

3. S. M. Rosen, Topologies of the Flesh (Ohio U. Press, Athens, OH, 2006).

4. S. M. Rosen, The Self-Evolving Cosmos (World Scientific, 2008).

5. O. Barfield, Saving of Appearances (Wesleyan U. Press, Middletown, CT, 1988).

6. L. Smolin, The Trouble with Physics (Houghton Mifflin, Boston, 2006).

7. P. Woit, Not Even Wrong (Basic Books, New York, 2006).

8. B. Greene, The Elegant Universe (W. W. Norton, New York, 1999).

9. M. Merleau-Ponty, "Eye and Mind," in The Primacy of Perception, J. M. Edie, ed. (Northwestern U. Press, Evanston, IL, 1964).

10. E. Husserl, Crisis of the European Sciences and Transcendental Phenomenology, D. Carr, trans. (Northwestern U. Press, Evanston, IL, 1936/1970).

11. M. Heidegger, Being and Time, J. Macquarrie, trans. (Harper and Row, New York, 1927/1962).

12. M. Merleau-Ponty, The Visible and the Invisible, A. Lingis, trans. (Northwestern U. Press, Evanston, IL, 1968).

13. P. Heelan, "Consciousness as Meaning-Maker: The Role of Consciousness in Science and Culture," paper presented at Toward a Science of Consciousness Conference, Budapest, Hungary, 23-26 July, 2007.

14. W. Heisenberg, "Grundlegende Voraussetzungen in der Physik der Elementarteilchen," in Martin Heidgegger zum siebzigsten Geburtstag (Festschrift), G. Neske, ed. (Neske, Pfullingen, 1959).

15. H. Weyl, Space-Time-Matter, H. Brose, trans. (Springer Verlag, Berlin, 1950).

16. J. Kockelmans, Ideas for a Hermeneutic Phenomenology of the Natural Sciences (Kluwer, Dordrecht, 2002).

17. P. Kurszberg, Critique and Totality (State U. of New York Press, Albany, NY, 1997).

18. P. Heelan, Space-Perception and the Philosophy of Science (U. of California Press, Berkeley, CA, 1983).

19. D. Bohm, Wholeness and the Implicate Order (Routledge and Kegan Paul, London, 1980).

20. S. Barr, Experiments in Topology (Dover, New York, 1964). 
21. M. Heidegger, "The Thinker as Poet," in Poetry, Language, Thought, A. Hofstadter, trans. (Harper and Row, New York, 1954/1971).

22. S. M. Rosen, Science, Paradox, and the Moebius Principle (State U. of New York Press, Albany, NY, 1994).

23. S. M. Rosen, "Pouring Old Wine Into a New Bottle," in The Interactive Field in Analysis, M. Stein, ed. (Chiron Publications, Wilmette, IL, 1995).

24. S. M. Rosen, "Wholeness as the Body of Paradox," J. Mind. Behav. 18, 391 (1997).

25. P. Ryan, Video Mind/Earth Mind (Peter Lang, New York, 1993).

26. R. Rucker, Geometry, Relativity, and the Fourth Dimension (Dover Books, New York, 1977).

27. R. Penrose, "Angular Momentum: An Approach to Combinatorial Space-Time," in Quantum Theory and Beyond, T. Bastin, ed. (Cambridge U. Press, Cambridge, 1971).

28. C. Musès, "Explorations in Mathematics," Impact of Science on Society 27, 67 (1977).

29. D. Applebaum, "Dirac Operators-From Differential Calculus to the Index Theorem," based on inaugural lecture at Nottingham Trent University, http://www.facct.ntu.ac.uk/staff/personal/dapplebaum/absconc.pdf (2000; accessed May 8, 2004; link currently dead).

30. C. Musès, "Applied Hypernumbers: Computational Concept," Appl. Math. and Comp. 3, 211 (1976).

31. F. A. M. Frescura and B. Hiley, "The Implicate Order, Algebras, and the Spinor," Found. Phys. 10, 7 (1980).

32. D. Hestenes, "Quantum Mechanics from Self-Interaction," Found. Phys. 15, 63 (1983).

33. H. Hu and M. Wu, "Spin as Primordial Self-Referential Process Driving Quanum Mechanics, Spacetime Dynamics and Consciousness," Neuroquant. 2, 41 (2004).

34. D. Hofstadter, Gödel, Escher, Bach: An Eternal Golden Braid (Basic Books, New York, 1979).

35. C. Musès, "Hypernumber and Metadimension Theory," J. of Cons. Stud. 1, 29 (1968).

36. M. Heidegger, "Time and Being," in On Time and Being, J. Stambaugh, trans. (Harper and Row, New York, 1962/1972).

37. H. Pagels, Perfect Symmetry (Bantam, New York, 1985). 